\title{
Three new active stars at high galactic latitudes ${ }^{\star}$
}

\author{
J. Martí1 ${ }^{1}$, J. M. Paredes ${ }^{2}$, J. L. Garrido ${ }^{1}$, and P. Luque-Escamilla ${ }^{3}$ \\ 1 Departamento de Física, EPS, Universidad de Jaén, Virgen de la Cabeza 2, 23071 Jaén, Spain \\ e-mail: [jmarti;jlg]@ujaen.es \\ 2 Departament d'Astronomia i Meteorologia, Universitat de Barcelona, Av. Diagonal 647, 08028 Barcelona, Spain \\ e-mail: jmparedes@ub.edu \\ 3 Dpto. de Ing. Mecánica y Minera, EPS, Universidad de Jaén, Av. Madrid 35, 23071 Jaén, Spain \\ e-mail: peter@ujaen.es
}

Received 18 February 2004 / Accepted 7 May 2004

\begin{abstract}
We report the confirmation of three X-ray and radio stars at high galactic latitudes as a by-product of a search originally aiming to find new microquasars. The objects are the late type, optically bright stars HD 135743, CD-2412231 and HD 220338. Interestingly, the last of them has displayed, on time scales of years, what could be considered as a radio flaring behaviour and deserves follow up. The observational evidence gathered, at radio and optical wavelengths, suggests that these objects are likely new chromospherically active stars relatively close to the Sun.
\end{abstract}

Key words. stars: individual: HD 135743, HD 220338, CD-2412231 - radio continuum: stars - X-rays: stars

\section{Introduction}

The identification of new classes of galactic $\mathrm{X}$ and/or $\gamma$-ray sources is among the priorities in today's high energy Astrophysics. Important progress here is likely to be obtained from appropriate exploitation of modern surveys and databases available in different wavelength domains (X-ray, radio, optical, etc.). Their on-line public access adds even more advantages. We have tried to contribute to this goal by conducting extensive searches for new radio emitting X-ray binaries and microquasars in the Galactic Plane using survey data. Our results so far range from a fully successful identification in one case (the relativistic jet source LS 5039, Paredes et al. 2000) to other possible candidates at low galactic latitudes within $\left|b^{I I}\right| \leq 5^{\circ}$ (Paredes et al. 2002; Ribó et al. 2002a; Martí et al. 2004).

However, the mining of modern electronic surveys is likely to reveal many other interesting objects that, originally, one is not looking for. Such by-products of the search may nevertheless have their own interest in other fields of Astrophysics. The purpose of this paper is to report three of these non-targeted findings which, although not microquasars, are likely examples of new active binary systems, such as of RS Canis Venaticorum (RS CVn) type.

The stars studied in this paper are all located at high galactic latitudes $\left|b^{I I}\right| \geq 20^{\circ}$. This may seem in contradiction with the fact that most galactic jet sources initially targeted by our

* Tables 1,2 and 4 are only available in electronic form at http://www . edpsciences.org search are often found close to the Galactic Plane. In contrast, our motivation to scan higher latitudes was to look for galactic jet sources in the halo of the Galaxy whose existence is suggested by some recent findings (see e.g., Romero et al. 2004). For instance, the microquasar Scorpius X-1 has been shown to move on a galactic orbit that strongly resembles that of the oldest stars and globular clusters of the inner Galactic Halo (Mirabel \& Rodrigues 2003). Also, XTE J1118+480 is a nearby high-velocity black hole binary on a Galactic-Halo orbit as reported by Mirabel et al. (2001). Moreover, in a previous work it has been shown that the star LS 5039 is, in fact, a runaway microquasar escaping into the Halo at $150 \mathrm{~km} \mathrm{~s}^{-1}$ with respect to its own regional standard of rest (Ribó et al. 2002b).

In this context, we conducted a preliminary search at $\left|b^{I I}\right| \geq$ $20^{\circ}$ by exploring possible candidates taken from the list of galactic bright X-ray sources identified by Bauer et al. (2000). As a result, we have been able to confirm the stars HD 135743, CD-2412231 and HD 220338 as new X-ray and radio emitters. In the following, we will present all our observational evidence available showing the stars peculiar nature, as well as provide a first classification of them into a known category of active stars.

\section{Selection of candidates}

The Bauer et al. (2000) list contains a total of 45 X-ray sources from the ROSAT All-Sky Survey Bright Source Catalogue (RBSC) (Voges et al. 1999) that were cross-identified with radio counterparts from the NRAO VLA Sky Survey (NVSS) (Condon et al. 1998). We decided to study only those cases 
Table 3. Results of the VLA observations in 2002.

\begin{tabular}{|c|c|c|c|c|c|c|}
\hline Source name & $\begin{array}{l}\text { Radio position } \\
\qquad \text { (ICRS) }\end{array}$ & $\begin{array}{l}S_{20 \mathrm{~cm}} \\
(\mathrm{mJy})\end{array}$ & $\begin{array}{l}S_{6 \mathrm{~cm}} \\
(\mathrm{mJy})\end{array}$ & $\begin{array}{l}S_{3.5 \mathrm{~cm}} \\
(\mathrm{mJy})\end{array}$ & $\alpha_{6 \mathrm{~cm}}^{20 \mathrm{~cm}}$ & $\alpha_{3.5 \mathrm{~cm}}^{6 \mathrm{~cm}}$ \\
\hline HD 135743 & $\begin{array}{l}15^{\mathrm{h}} 17^{\mathrm{m}} 21^{\mathrm{s}} .193 \pm 0.001 \\
-19^{\circ} 00^{\prime} 59^{\prime} \cdot 26 \pm 0 !^{\prime} 01\end{array}$ & $5.57 \pm 0.15$ & $8.33 \pm 0.09$ & $6.87 \pm 0.11$ & $+0.74 \pm 0.05$ & $-0.35 \pm 0.04$ \\
\hline CD-2412231 & $\begin{array}{l}15^{\mathrm{h}} 41^{\mathrm{m}} 31^{\mathrm{s}} .209 \pm 0.004 \\
-25^{\circ} 20^{\prime} 36^{\prime \prime} \cdot 49 \pm 00^{\prime} 05\end{array}$ & $\leq 1.16$ & $1.17 \pm 0.06$ & $0.74 \pm 0.02$ & $\geq+0.0$ & $-0.8 \pm 0.1$ \\
\hline HD 220338 & $\begin{array}{c}23^{\mathrm{h}} 23^{\mathrm{m}} 01^{\mathrm{s}} .17 \pm 0.01 \\
-06^{\circ} 35^{\prime} 43^{\prime \prime} .8 \pm 00^{\prime} 1\end{array}$ & $\leq 0.83$ & $0.27 \pm 0.04$ & $0.36 \pm 0.03$ & $\geq-1.3$ & $+0.5 \pm 0.3$ \\
\hline
\end{tabular}

where a likely identification with a bright catalogued star has been proposed. Among them, the Bauer et al. (2000) list contains well known "active stars" such as RS CVn or cataclysmic variables. These already known objects were not considered in our work based on consultation of the SIMBAD database. Instead, we focused our attention on anonymous stellar objects at $\left|b^{I I}\right| \geq 20^{\circ}$ with a ROSAT hardness ratio (HR1) higher than about 0.9, as typical of X-ray binary spectra (Motch et al. 1998). Only two poorly known stars were retained at this point, namely HD 135743 and CD-2412231. We also decided to include HD 220338 in our target list in spite of its lower value of $H R 1$. The reason at that time was its high NVSS flux density (13.4 mJy) with practically no reference being found in the SIMBAD database.

The basic parameters of the three stars studied in this work are listed in Table 1. The extinction and distance to each of them has been computed based on the observed apparent magnitude and spectral type (see Sect. 5). The X-ray luminosity, in the $0.1-2.4 \mathrm{keV}$ band, is derived from the ROSAT PSPC count rate. It has been computed assuming a photon power index of $\Gamma=2.0$ and using the tool PIMMSv3.2d, originally developped by Mukai (1993).

\section{Radio observations and results}

The selected stars were observed with the Very Large Array (VLA) of the National Radio Astronomy Observatory (NRAO) on 2002 July 16th and 17th. At that time, the VLA was in its $\mathrm{B}$ configuration with maximum baselines extending over $11 \mathrm{~km}$. The sources were observed at the frequencies of $1.49,4.91$ and $8.46 \mathrm{GHz}(20,6$ and $3.5 \mathrm{~cm}$, respectively) using two IF pairs of $50 \mathrm{MHz}$ bandwidth each. HD 220338 was observed alone during the first run while the second run was devoted to HD 135743 and CD-2412231. The primary flux density calibrators used were 3C 286 and 3C 48.

As phase calibrators, we observed 1507-168, 1522-275 and 2323-032. Their bootstrapped flux densitites at each wavelength, typically accurate to $\pm 0.01 \mathrm{Jy}$, are given in Table 2 together with the detailed log. The observed radio properties of the three candidates are listed in Table 3, which contains the source name, its radio position, the different flux densities $\left(S_{v}\right)$ and spectral indices $\left(S_{v} \propto v^{\alpha}\right)$ between them. Maps are shown

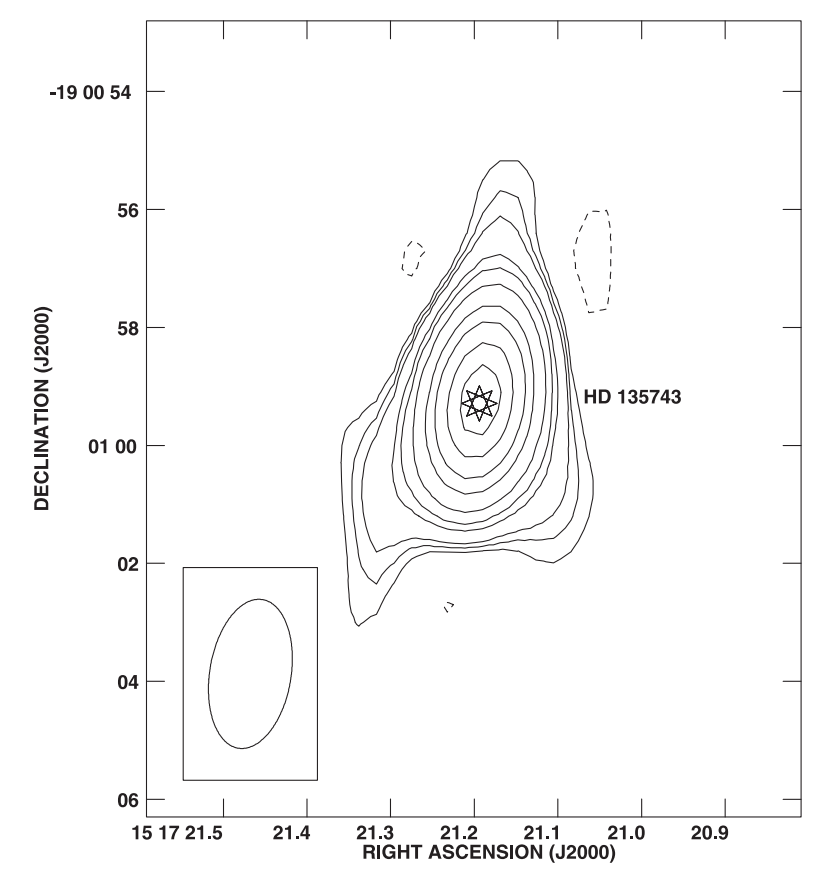

Fig. 1. VLA snapshot of HD 135743 at the $6 \mathrm{~cm}$ wavelength where this radiostar was detected with higher signal-to-noise ratio. The star symbol indicates the position of the optical star as expected from our proper motion determination. The agreement between the optical and radio position is excellent within less than $0 .{ }^{\prime} 1$. This snapshot was computed with natural weight and its synthesized beam is 2 ". $55 \times 1$ ". 38 (shown at the bottom left corner), with position angle of -9.23 . Contours are $-4,4,6,8,15,20,30,50,70,100$ and 130 times the rms noise of $0.054 \mathrm{mJy}^{\mathrm{beam}}{ }^{-1}$. The extensions of the contours are artifacts from the deconvolution procedure.

in Figs. 1-3 at the wavelengths with higher signal-to-noise ratio. Our VLA observations provide a significant increase in accuracy with respect to the NVSS radio positions, which in some cases had uncertainties of up to $\sim 7^{\prime \prime}$.

\section{Optical astrometry}

To check the positional coincidence of the stars and their radio counterparts as proposed by Bauer et al. (2000), we performed an analysis of optical astrometry from several catalogs. 


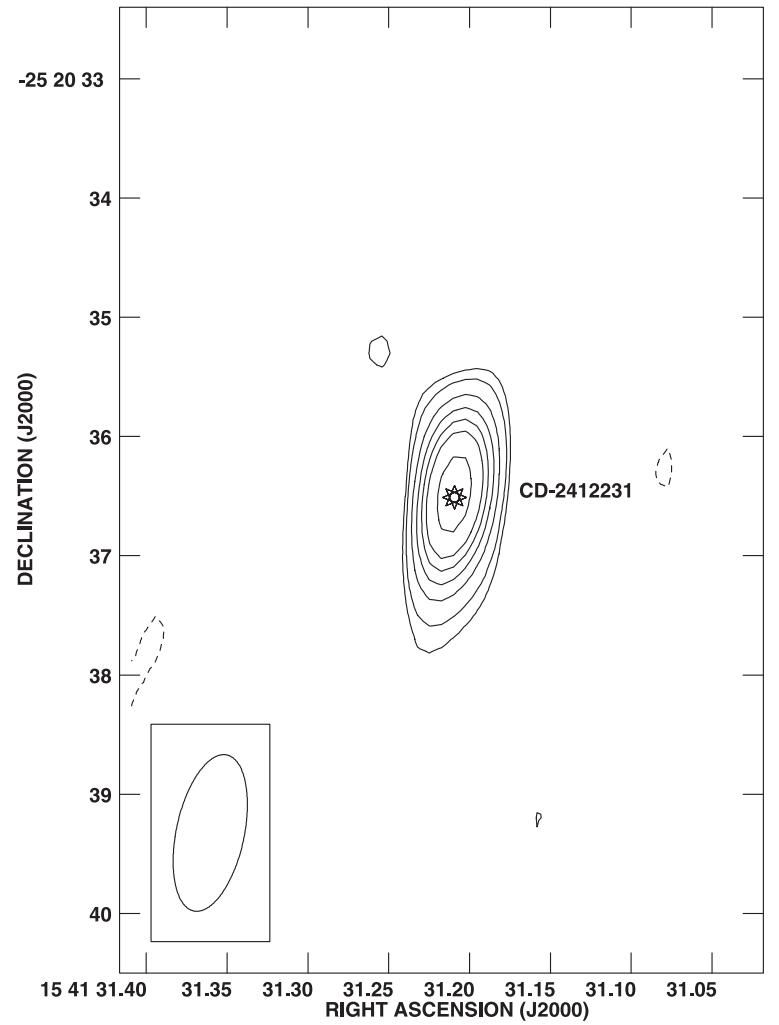

Fig. 2. VLA map of CD-2412231 with uniform weight at the $3.5 \mathrm{~cm}$ wavelength where this radiostar was detected with higher signal-tonoise ratio and angular resolution. The star symbol indicates the position of the optical star as expected from our proper motion determination. The agreement between the optical and radio position is again excellent within less than 0 !' 1 . The synthesized beam is 1 '. $34 \times 0$ '.57 (bottom left corner), with position angle of -11 .97. Contours are -3 , $3,4,6,8,10,12$ and 16 times the rms noise of $0.041 \mathrm{mJy}^{\text {beam }}{ }^{-1}$.

In Table 4 we have listed the different positions for the observed stars. In some cases, the time baseline spans more than a century which allows a reliable determination of the apparent proper motion (Figs. 4-6). The catalogs used are: AC2000.2 (Urban et al. 1998); CPC2 (Zacharias et al. 1999); USNO A2.0 (Monet et al. 1999); GSC 2.2.1 (STScI \& Oss. Ast. di Torino 2001); Tycho 2 (Hog et al. 2000); UCAC1 (Zacharias et al. 2000); PPM South (Bastian \& Roeser 1993); SPM (Platais et al. 1998); TAC 2.0 (Zacharias \& Zacharias 1999); CMC (Carlsberg Meridian Catalogs 1999).

Using the proper motions obtained in Table 5, the positions of the three stars at the epoch of our VLA observations (2002.54) have the following ending figures in right ascension and declination (both expressed in arcseconds): HD 135743

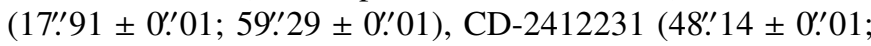

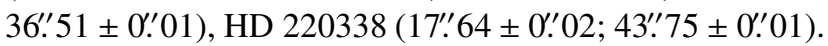

These ICRS positions correspond to the star symbols plotted on the VLA contour maps shown in Figs. 1-3. In all cases, the agreement between the optical and radio position is well within the sub-arcsecond astrometric error. Therefore, the identification of these objects as radio stars appears very reliable to us.

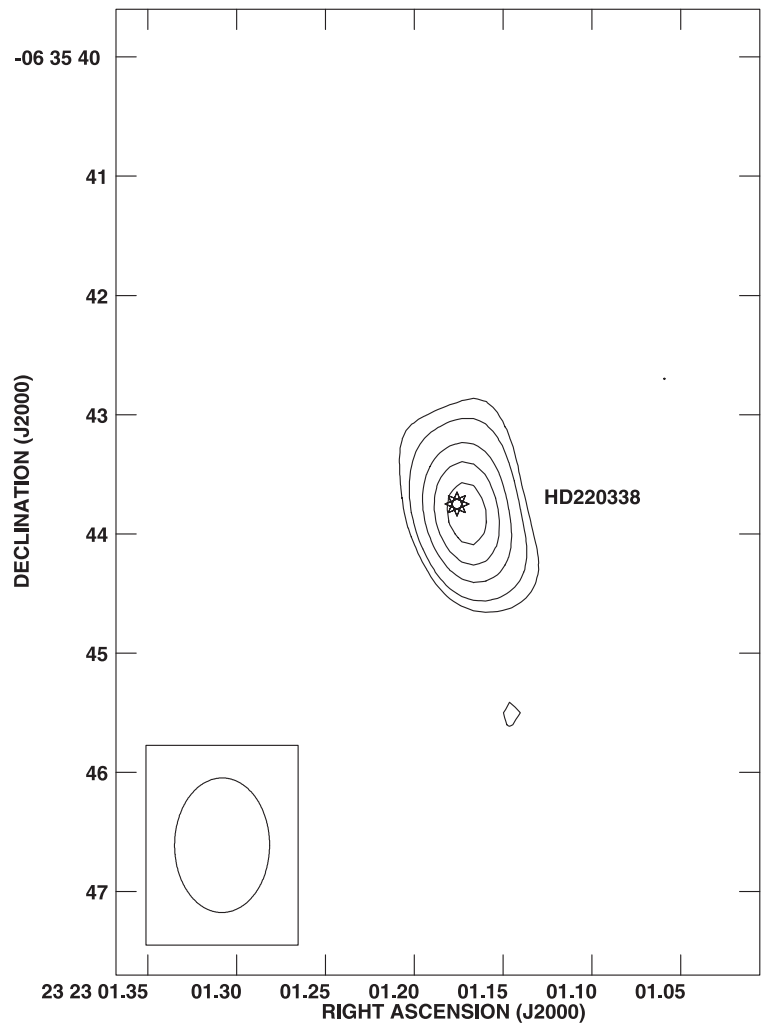

Fig. 3. VLA map of HD 220338 with natural weight at the $3.5 \mathrm{~cm}$ wavelength where this radiostar was detected with higher signal-tonoise ratio and angular resolution. The star symbol indicates the position of the optical star as expected from our proper motion determination. The agreement between the optical and radio position is good within $0{ }^{\prime} 1$. The synthesized beam is $11^{\prime \prime} 13 \times 00^{\prime} 80$ (bottom left corner), with position angle of -0.20 . Contours are $-3,3,4,6,8$ and 10 times the rms noise of $0.026 \mathrm{mJy}^{\text {beam }^{-1}}$.

\section{Optical spectroscopy}

With the idea to better constrain the nature of the three radio stars, we secured first low resolution optical spectroscopy for all of them. HD 135743 and CD-2412231 were observed on 2002 March 23 with the Danish 1.54 m telescope + DFOSC of the European Southern Observatory (ESO) at La Silla (Chile). HD 220338 was observed on 2002 May 29 with the $2.2 \mathrm{~m}$ telescope + CAFOS of the Centro Astronómico Hispano Alemán (CAHA) at Calar Alto ${ }^{1}$ observatory (Spain).

The low resolution spectra of HD 135743, CD-2412231 and HD 220338 are presented in Fig. 7. They were reduced using the IRAF package of NOAO including bias subtraction, spectroscopic flat fielding, optimal extraction and wavelength calibration. The Calar Alto spectrum, obtained at a very high air mass, is in arbitrary flux units as it could not be flux calibrated.

Higher resolution spectra were also acquired for all stars during 2003 May 14 to 17 and processed in a similar way. We used the Intermediate Dispersion Spectrograph (IDS) at the

${ }^{1}$ Based on observations collected at the German-Spanish Astronomical Center, Calar Alto, operated jointly by Max-Planck Institut für Astronomie and Instituto de Astrofísica de Andalucía (CSIC). 

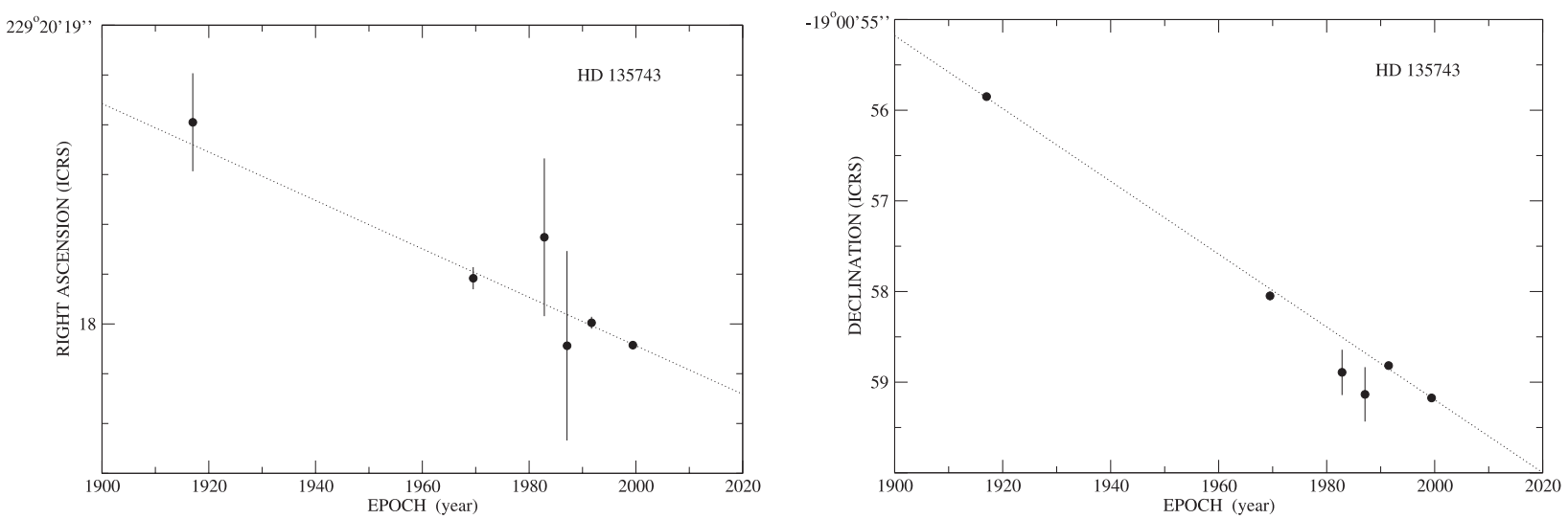

Fig. 4. Changes in the ICRS right ascension and declination of the radiostar HD 135743 as evidenced from historical positions in the different optical catalogs of Table 4. The dotted lines represent least squares fits whose respective slopes give the star proper motion listed in Table 5 . Error bars not shown are smaller than the symbol size.
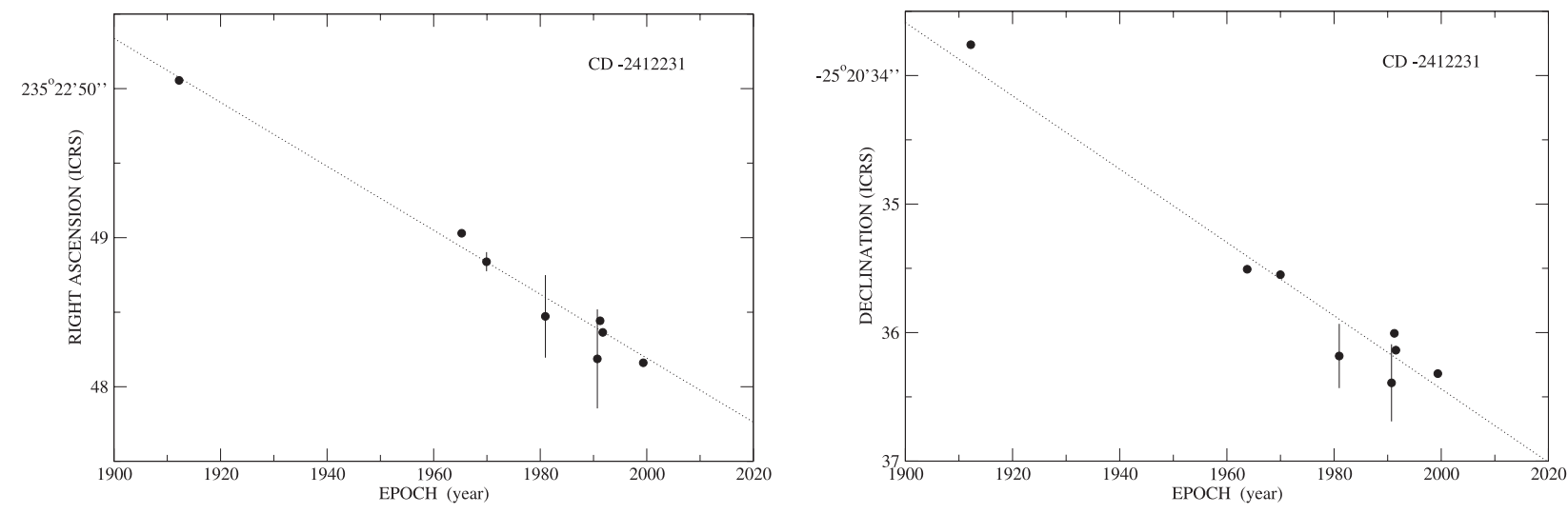

Fig. 5. The same kind of plot as in Fig. 4 for the radiostar CD-2412231.
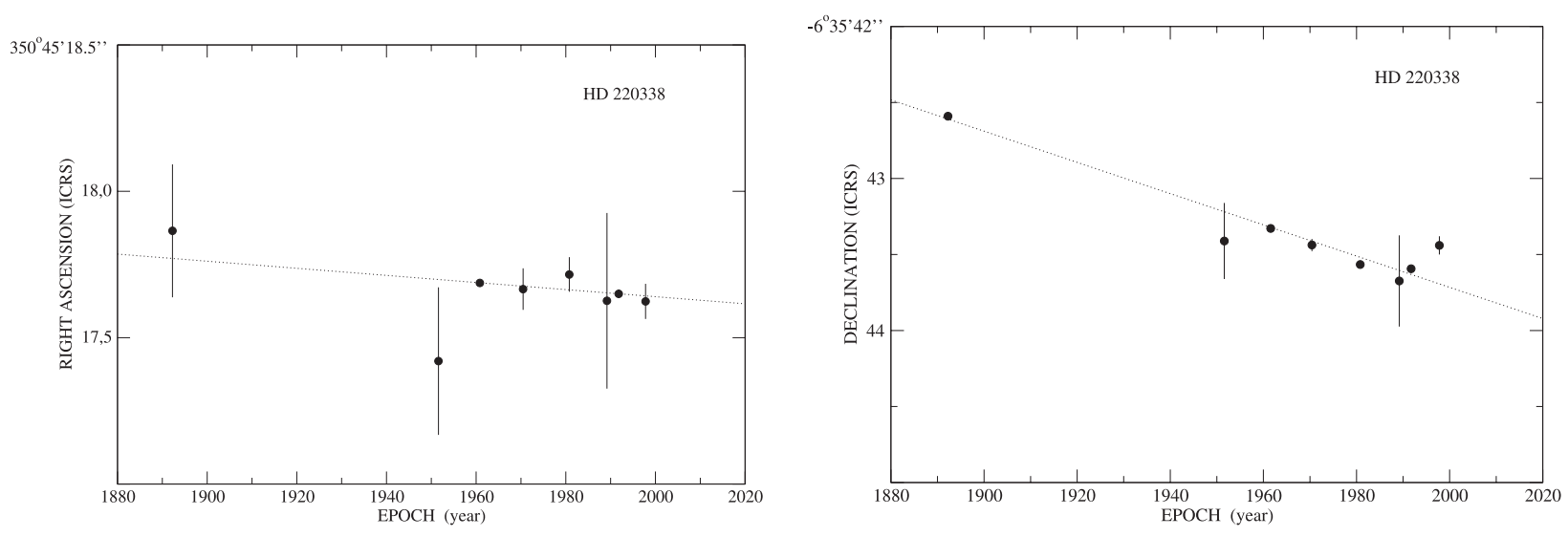

Fig. 6. The same kind of plot as in Fig. 4 for the radiostar HD 220338.

$2.5 \mathrm{~m}$ Isaac Newton Telecope (INT) in Canary Islands (Spain). The INT spectra cover the range 3800-4500 $\AA$ and are suitable for accurate spectral classification and radial velocity determination. In Fig. 8 we show in detail the region of the $\mathrm{H}$ and $\mathrm{K}$ absorption lines of $\mathrm{Ca}$ II, which clearly have central cores in emission. In Figs. 9-11, we compare the INT spectra, in the wavelength range 4000-4500 , with different stellar templates for spectral type determination. It is from this comparison that we obtained the spectral types listed in Table 1. For a binary system, the proposed classification should mostly reflect the spectral type of the binary component dominating the optical luminosity.

\section{Discussion}

Our search for microquasars based on the Bauer et al. (2000) list did not reveal new findings associated with optically bright stars. This immediately suggests that such objects at high galactic latitudes are likely not numerous. 
Table 5. Optical positions and proper motions.

\begin{tabular}{|c|c|c|}
\hline Star & Right ascension (ICRS) & Declination (ICRS) \\
\hline \multirow{2}{*}{ HD 135743} & $229^{\circ} 20^{\prime} 17^{\prime \prime} \cdot 93 \pm 0.0^{\prime} 01$ & $-19^{\circ} 00^{\prime} 59^{\prime} \cdot 19 \pm 0 . .^{\prime} 01$ \\
\hline & $-\left(8.1 \pm 1.1{\left.\mathrm{mas} \mathrm{yr}^{-1}\right) \times(t-2000)}^{-1}\right.$ & $-\left(40.2 \pm 0.5 \mathrm{mas} \mathrm{yr}^{-1}\right) \times(t-2000)$ \\
\hline \multirow[t]{2}{*}{ CD-2412231 } & $235^{\circ} 22^{\prime} 48^{\prime \prime} \cdot 19 \pm 00^{\prime} 01$ & $-25^{\circ} 20^{\prime} 36^{\prime \prime} 44 \pm 00^{\prime} 01$ \\
\hline & $-\left(21.5 \pm 0.1 \mathrm{mas} \mathrm{yr}^{-1}\right) \times(t-2000)$ & $-\left(28.5 \pm 0.3\right.$ mas $\left.\mathrm{yr}^{-1}\right) \times(t-2000)$ \\
\hline \multirow[t]{2}{*}{ HD 220338} & $350^{\circ} 45^{\prime} 17^{\prime \prime} 64 \pm 00^{\prime} 02$ & $-06^{\circ} 35^{\prime} 43^{\prime \prime} 72 \pm 0 .{ }^{\prime} 01$ \\
\hline & $-\left(1.2 \pm 0.5 \mathrm{mas} \mathrm{yr}^{-1}\right) \times(t-2000)$ & $-\left(10.3 \pm 0.2 \mathrm{mas} \mathrm{yr}^{-1}\right) \times(t-2000)$ \\
\hline
\end{tabular}
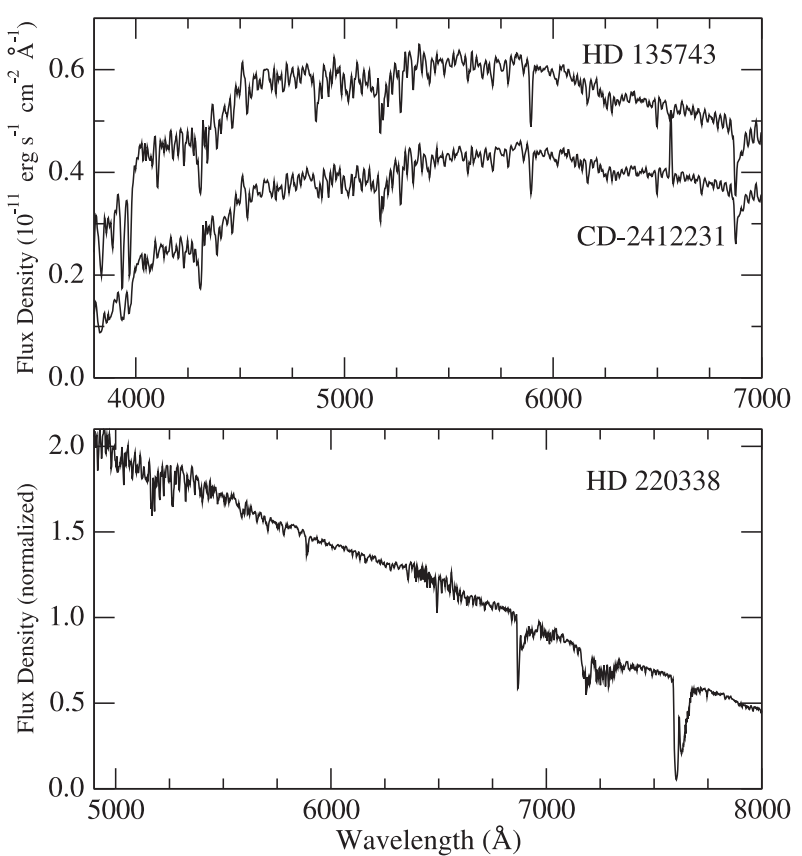

Fig. 7. Top: low resolution optical spectrum of HD 135743 and CD-2412231 taken with DFOSC at the $1.54 \mathrm{~m}$ ESO telescope. $\mathrm{H} \alpha$ in emission is clearly detected in CD-2412231. Bottom: low resolution optical spectrum of HD 220338 taken with CAFOS at the $2.2 \mathrm{~m}$ CAHA telescope. $\mathrm{H} \alpha$ in emission is marginally detected.

Independently of this, our main goal here is to discuss the main properties of the three new active stars confirmed as a by-product of our search. All of them displayed emission cores in the $\mathrm{Ca}$ II $\mathrm{H}$ and $\mathrm{K}$ lines and even two of them had $\mathrm{H} \alpha$ in emission. These facts are typical indicators of chromospherically active stars, a category which includes RS CVns, Algols, AM Her, cataclysmic variables, etc. (Hilditch 2001; Güdel 2002). The X-ray luminosities of $10^{30}-10^{31} \mathrm{erg} \mathrm{s}^{-1}$, in the ROSAT X-ray band, derived from the distances estimated spectroscopically are significantly lower than in typical X-ray binaries. For instance, the luminosity range of RS CVn systems is usually within $\sim 10^{29}-10^{31.5} \mathrm{erg} \mathrm{s}^{-1}$ (Dempsey et al. 1993).

In addition, there is a well know correlation between quiescent radio and X-ray luminosities of magnetically active stars of the type $L_{\mathrm{R}} \propto L_{\mathrm{X}}^{1.0-1.3}$ (see e.g., Benz \& Güdel 1994). Using the ROSAT count rates for our sources toghether with

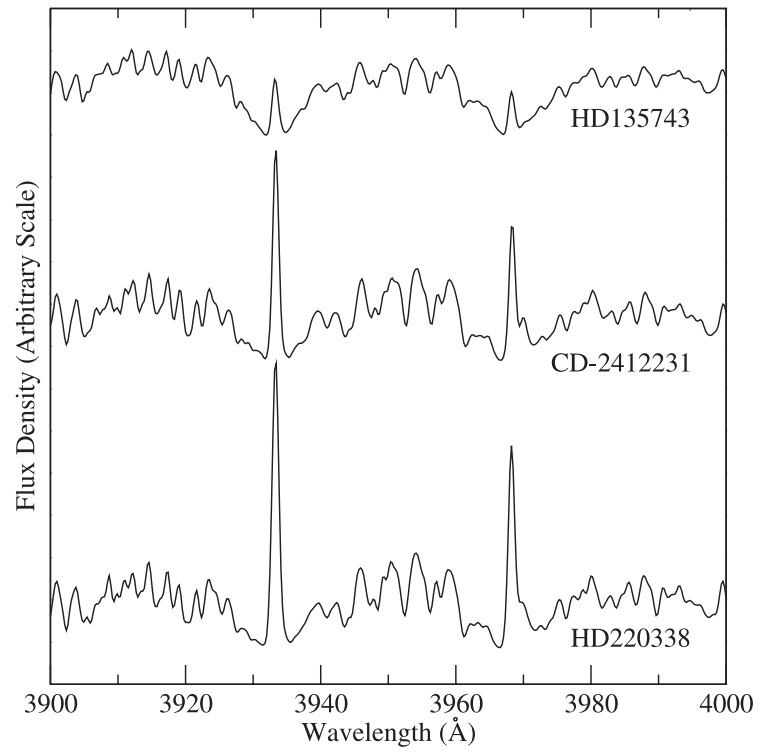

Fig. 8. INT+IDS spectra of HD 135743, CD-2412231 and HD 220338 showing in detail the core emission components in the CaII $\mathrm{H}$ and $\mathrm{K}$ absorption lines.

our VLA radio flux densities, all of them appear to be consistent with such a correlation. This reinforces our suggestion that we are dealing with the kind of magnetically active cool stars quoted above, whose emission mechanism is of a non-thermal nature.

In the following subsections, we discuss each source individually.

\section{1. $H D 135743$}

We have been able to confirm the binary nature of HD 135743 after obtaining the cross-correlation function of its spectrum with a G3 V template. The result, shown in Fig. 12, clearly displays two well separated maxima evolving from night to night. This fact implies that HD 135743 is a new double-lined spectroscopic binary (SB2), whose two components are nondegenerate stars with probably a very similar spectral type. The tomographic separation of the spectra and full radial velocity curves is beyond the scope of this paper and will require more extensive observations. 


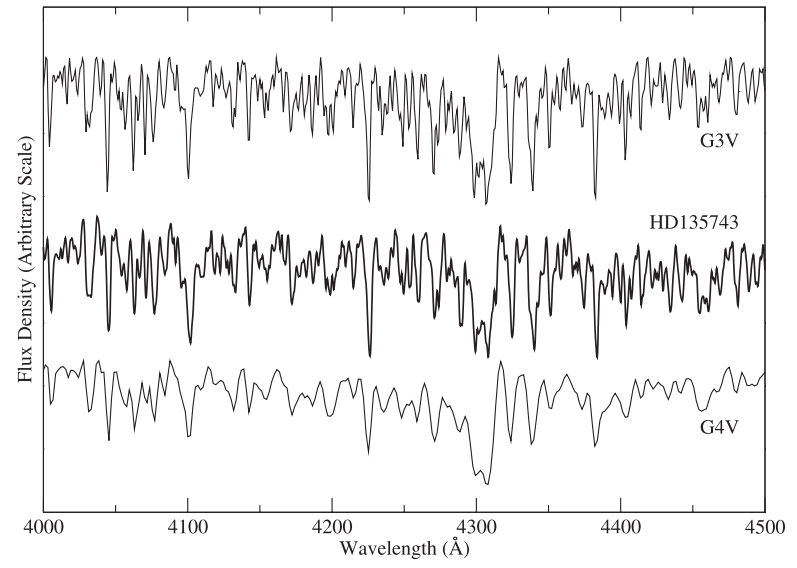

Fig. 9. INT+IDS spectrum of HD 135743 plotted against different stellar templates. The comparison suggests a spectral type G3 V for this star.

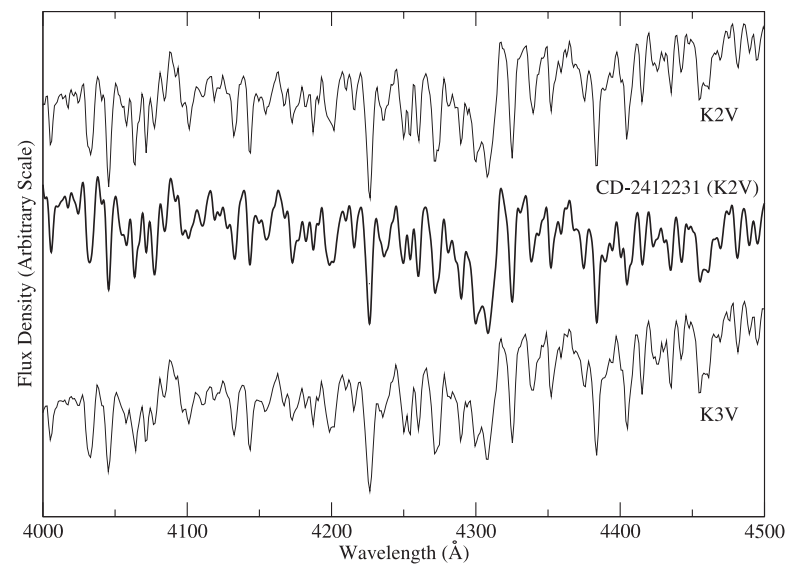

Fig. 10. INT+IDS spectrum of CD-2412231 plotted against different stellar templates. The comparison suggests a spectral type K2 V for this star.

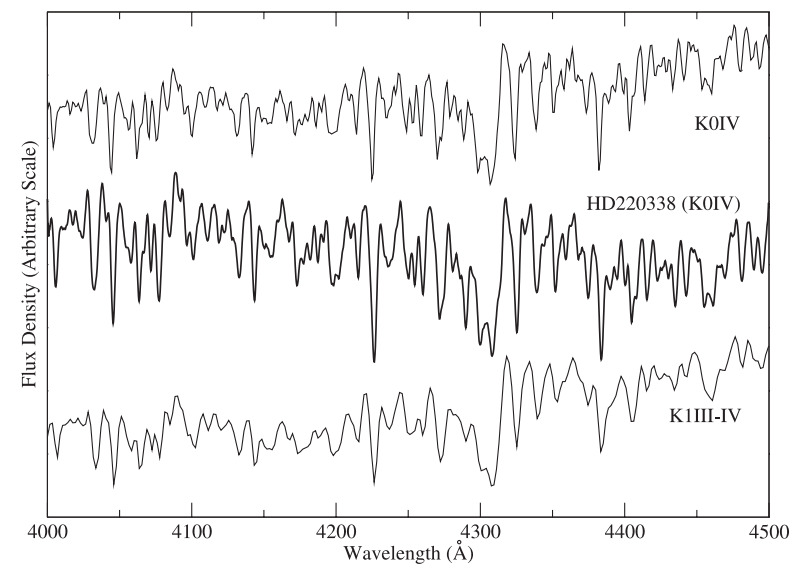

Fig. 11. INT+IDS spectrum of HD 220338 plotted against different stellar templates. The comparison suggests a spectral type K0 IV for this star.

The observed radio spectrum in Table 3 is consistent with optically thin synchrotron emission at the shorter wavelengths. HD 135743 is probably a chromospherically active binary with an orbital period of a few days.

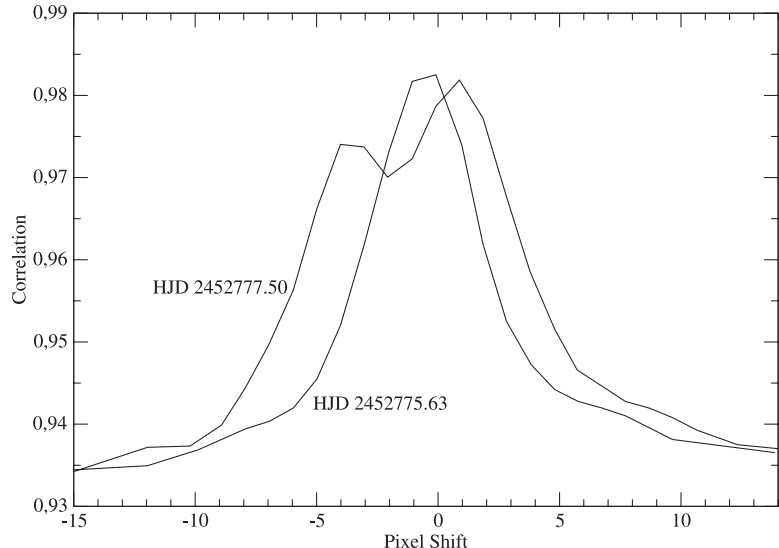

Fig. 12. Result of the cross-correlation of a typical INT spectrum of HD 135743 with a G3 V template using the FXCOR task of IRAF. The appearance of two maxima at some times is a clear indicator that this object is a double-lined spectroscopic binary. Both components are likely late type stars.

\section{2. $C D-2412231$}

We have not been able to prove the binary nature of this object, which could be a single star with chromospheric activity. This statement is based on the fact that our INT observations did not reveal convincing radial velocity variations during four consecutive nights. However, the possibility of a binary system with an orbital period of few hours such as an AM Her system (hence not well sampled with the INT) could not be strictly ruled out.

The observed radio spectrum is clearly consistent with optically thin synchrotron emission between 6 and $3.5 \mathrm{~cm}$, with its peak probably between 20 and $6 \mathrm{~cm}$. Hints of significant circular polarization are also present in difference maps made using the $R R$ and $L L$ cross-correlation products of the VLA.

\subsection{HD 220338}

This radio star is remarkable because it has undergone strong radio variability at least on time scales of years. This statement is based on the comparison between the NVSS and our own radio observations as shown in Fig. 13. In 1993, HD 220338 was detected in the NVSS with $S_{20 \mathrm{~cm}}=13 \mathrm{mJy}$. In contrast, our 2002 VLA observations provided just an upper limit of $S_{20 \mathrm{~cm}} \leq 0.83 \mathrm{mJy}$, thus implying a variation by a factor of $\gtrsim 16$. This factor would increase to $\sim 30$ by extrapolating the flux densities measured at 6 and $3.5 \mathrm{~cm}$ (see Table 3 ). These results are suggestive that HD 220338 behaves as a radio flaring source, although radio variability on shorter time scales still needs to be investigated.

Our INT spectroscopy successfully revealed Doppler variations in the radial velocity, thus indicating that HD 220338 is a binary system. However, not enough data is yet available to provide a consistent orbital solution. Contrary to the HD 135743 case, the cross-correlation functions obtained are always single peaked and we have found no trace of an optical companion to the proposed K0 IV star, even when looking at the individual $\mathrm{H}$ and $\mathrm{K}$ emission lines. 

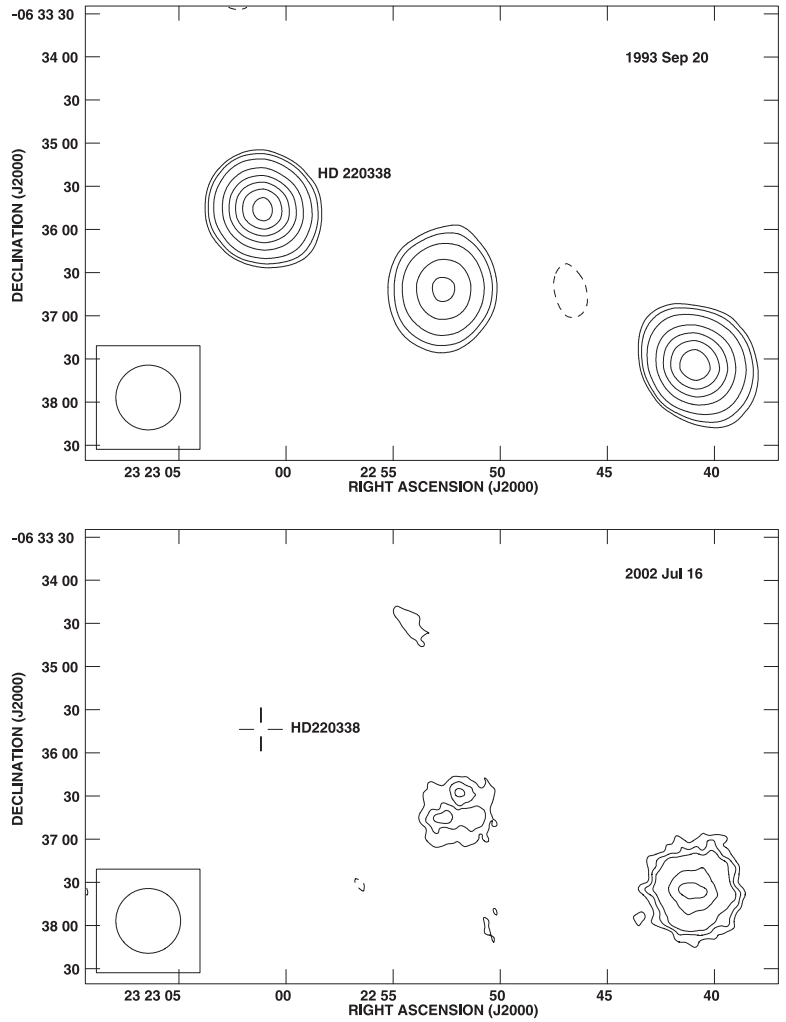

Fig. 13. Evidence for strong radio variability of HD 220338 at the $20 \mathrm{~cm}$ wavelength during a time scale of years. The top panel is the image from the NVSS survey taken in 1993 September 20th and convolved with a circular restoring beam of $45^{\prime \prime}$ (bottom left corner). The NVSS contours shown are $-3,3,4,6,10,14,18,22$ and 26 times $0.45 \mathrm{mJy}^{\text {beam }}{ }^{-1}$, the rms noise. The bottom panel is our VLA image of the same region as we observed it at the same wavelength in 2002 July 16 . This map has been computed with a $u v$ taper of $20 \mathrm{k} \lambda$ and the same NVSS restoring beam for easier comparison between the two epochs. Contours here are $-4,4,6,8,15,20$ times $0.3 \mathrm{mJy}$ beam $^{-1}$, the rms noise. The cross with a gap indicates the position of HD 220338 not detected during the second epoch at $20 \mathrm{~cm}$.

The radio spectrum observed in 2002 displays a positive spectral index although with high uncertainty. A thermal emission mechanism (e.g. from a wind) is not likely to explain the strong year variability observed in this source. Therefore, the possiblity of a self-absorbed synchrotron spectrum is a conceivable alternative.

The subgiant spectral type, ROSAT hardness ratio, Doppler variations and indicators of chromospheric activity suggest that this could be a new RS CVn binary.

\section{Conclusions}

We have reported the confirmation of three new radio and X-ray stars at high galactic latitudes, namely HD 135743, CD-2412231 and HD 220338. The original motivation for this work was the search for microquasar systems above the Galactic Plane. Although none was found, the objects reported here will increase the known population of nearby chromospherically active stars.

The proposed binary nature of HD 135743 and HD 220338 is based on spectroscopic observations. In addition, HD 220338 has been shown to display a dramatic radio flaring behaviour over the years. Further observations are in progress to clarify the proposed nature of these objects and to obtain well sampled radial velocity curves.

Acknowledgements. The authors acknowledge partial support by DGI of the Ministerio de Ciencia y Tecnología (Spain) under grant AYA2001-3092. J.M. has been also aided in this work by an Henri Chrétien International Research Grant administered by the American Astronomical Society and is also supported by the Junta de Andalucía (Spain) under project FQM322. This research has made use of the NASA's Astrophysics Data System Abstract Service and of the SIMBAD database, operated at CDS, Strasbourg, France. The National Radio Astronomy Observatory is a facility of the National Science Foundation operated under cooperative agreement by Associated Universities, Inc. in the USA The INT is operated on the island of La Palma by the Royal Greenwich Observatory in the Spanish Observatorio del Roque de Los Muchachos of the Instituto de Astrofísica de Canarias. The authors also thank F. Comerón (ESO) for kindly obtaining some of the optical spectra reported in this paper.

\section{References}

Bauer, F. E., Condon, J. J., Thuan, T. X., et al. 2000, ApJS, 129, 547 Bastian, U., \& Roeser, S. 1993, Catalogue of Positions and Proper Motions - South, Astronomisches Rechen-Institut, Heidelberg

Benz, A. O., \& Güdel, M. 1994, A\&A, 285, 621

Carlsberg Meridian Catalogs, Number 1-11, Copenhagen University Obs., Royal Greenwich Obs., and Real Instituto y Observatorio de la Armada en San Fernando

Condon, J. J., Cotton, W. D., Greisen, E. W., et al. 1998, AJ, 115, 1693

Dempsey, R. C., Linsky, J. L., Fleming, T. A., \& Schmitt, J. H. M. M. 1993, ApJS, 86, 599

Güdel, M. 2002, ARA\&A, 40, 217

Hilditch, R. W. 2001, An Introduction to Close Binary Stars (Cambridge University Press)

Hog, E., Fabricius, C., Makarov, V. V., et al. 2000, A\&A, 355, L27

Martí, J., Paredes, J. M., Bloom, J. S., et al. 2004, A\&A, 413, 309

Mirabel, I. F., Dhawan, V., \& Mignami, R. P. 2001, Nature, 413, 139

Mirabel, I. F., \& Rodrigues, I. 2003, A\&A, 398, L25

Monet, D. G., Bird, A., Canzian, B., et al. 1999, USNO-A2.0 CD-ROM (Washington DC: US Naval Observatory)

Motch, C., Guillout, P., Haberl, F., et al. 1998, A\&AS, 132, 341

Mukai, K. 1993, Legacy, 3, 21

Paredes, J. M., Martí, J., Ribó, M., \& Massi, M. 2000, Science, 288, 2340

Paredes, J. M., Ribó, M., \& Martí, J. 2002, A\&A, 394, 193

Platais, I., Girard, T. M., Kozhurina-Platais, V., et al. 1998, AJ, 116, 2556

Reig, P., Ribó, M., Paredes, J. M., \& Martí, J. 2003, A\&A, 405, 285

Ribó, M., Ros, E., Paredes, J. M., Massi, M., \& Martí, J. 2002a, A\&A, 394, 983

Ribó, M., Paredes, J. M., Romero, G. E., et al. 2002b, A\&A, 384, 954

Romero, G. E., Grenier, I. A., Kaufman Bernadó, M. M., et al. 2004, The INTEGRAL Universe, Proc. 5th INTEGRAL Workshop, in press [arXiv: astro-ph/0402285]

Space Telescope Science Institute (STScI) and Osservatorio Astronomico di Torino, 2001, The Guide Star Catalogue, Version 2.2.01

Urban, S. E., Corbin, T. E., Wycoff, G. L., et al. 1998, AJ, 115, 1212

Voges, W., Aschenbach, B., Boller, Th., et al. 1999, A\&A, 349, 389

Zacharias, N., \& Zacharias, M. I. 1999, AJ, 118, 2503

Zacharias, N., Zacharias, M. I., \& De Vegt, C. 1999, AJ, 117, 2895

Zacharias, N., Urban, S. E., Zacharias, M. I., et al. 2000, AJ, 120, 2131 


\section{Online Material}


J. Martí et al.: Three new active stars at high galactic latitudes, Online Material $p 2$

Table 1. List of targets.

\begin{tabular}{ccccccccc}
\hline \hline Source name & $\begin{array}{c}\text { Galactic } \\
\text { coordinates }\end{array}$ & Spectral type & $V$ & $B-V$ & $E(B-V)$ & $A_{\mathrm{V}}$ & $\begin{array}{c}\text { Estimated } \\
\text { distance }\end{array}$ & $\begin{array}{c}L_{\mathrm{X}}(0.1-2.4 \mathrm{keV})^{*} \\
\left(\mathrm{erg} \mathrm{s}^{-1}\right)\end{array}$ \\
\hline HD 135743 & $344^{\circ} .37,+31^{\circ} .87$ & G3 V & 9.40 & +0.80 & 0.15 & 0.45 & $83 \mathrm{pc}$ & $8 \times 10^{30}$ \\
CD-2412231 & $344^{\circ} .67,+23.43$ & K2 V & 9.88 & +0.82 & 0.19 & 0.56 & $37 \mathrm{pc}$ & $2 \times 10^{30}$ \\
HD 220338 & $73^{\circ} .27,-60^{\circ} 63$ & K0 IV & 9.09 & +1.09 & $\simeq 0.0$ & $\simeq 0.0$ & $150 \mathrm{pc}$ & $2 \times 10^{31}$ \\
\hline
\end{tabular}

${ }^{*}$ Unabsorbed X-ray luminosity.

Table 2. Log of the VLA observations.

\begin{tabular}{cccccc}
\hline \hline Epoch date & Julian Day & Configuration & $\begin{array}{c}\text { Wavelengths } \\
(\mathrm{cm})\end{array}$ & Phase calibrator & $\begin{array}{c}\text { Bootstrapped flux densities } \\
(\mathrm{Jy})\end{array}$ \\
\hline 2002 July 16 & 2452471.95 & B & $20,6,3.5$ & $2323-032$ & $0.81,0.88$ and 0.94 \\
2002 July 17 & 2452472.58 & B & $20,6,3.5$ & $1507-168$ & $3.04,2.47$ and 1.60 \\
& & & & $1522-275$ & $1.16,1.44$ and 2.07 \\
\hline
\end{tabular}


J. Martí et al.: Three new active stars at high galactic latitudes, Online Material p 3

Table 4. Record of historical optical astrometry.

\begin{tabular}{|c|c|c|c|c|}
\hline Star & $\begin{array}{l}\text { Epoch } \\
\text { (year) }\end{array}$ & Right ascension (ICRS) & Declination (ICRS) & Catalog \\
\hline & & $229^{\circ} 20^{\prime}$ & $-19^{\circ} 00^{\prime}$ & \\
\hline \multirow[t]{9}{*}{ HD 135743} & 1917.005 & $18^{\prime \prime} 675 \pm 0 . \prime 164$ & $55^{\prime \prime} .85 \pm 0.037$ & $\mathrm{AC} 2000.2$ \\
\hline & 1969.532 & $18^{\prime \prime} 153 \pm 0.0^{\prime} 037$ & - & $\mathrm{CPC} 2$ \\
\hline & 1969.498 & - & $58{ }^{\prime \prime} 048 \pm 0.049$ & $"$ \\
\hline & 1982.843 & $18^{\prime \prime} 290 \pm 0.264$ & $58^{\prime \prime} 892 \pm 0.250$ & USNO A 2.0 \\
\hline & 1987.090 & $17^{\prime \prime} 927 \pm 0.317$ & $59 . ' 134 \pm 0.300$ & GSC 2.2.1 \\
\hline & 1991.69 & $18^{\prime \prime} 004 \pm 0 !^{\prime} 020$ & & Tycho 2 \\
\hline & 1991.47 & - & $58^{\prime \prime} 817 \pm 0.024$ & $"$ \\
\hline & 1999.426 & $177^{\prime \prime} 929 \pm 0 . \prime 014$ & $59 ! 174 \pm 0 !^{\prime} 01$ & UCAC1 \\
\hline & & $235^{\circ} 22^{\prime}$ & $-25^{\circ} 20^{\prime}$ & \\
\hline \multirow[t]{12}{*}{ CD-2412231 } & 1912.212 & $50{ }^{\prime} 055 \pm 00^{\prime} 008$ & $33^{\prime \prime} \cdot 76 \pm 0.022$ & AC2000.2 \\
\hline & 1965.27 & $49 ! .029 \pm 00^{\prime} 011$ & - & PPM South \\
\hline & 1963.79 & - & $35^{\prime \prime} .507 \pm 0.010$ & $"$ \\
\hline & 1969.929 & $48{ }^{\prime \prime} 839 \pm 00^{\prime} 064$ & - & $\mathrm{CPC} 2$ \\
\hline & 1969.999 & - & $35^{\prime \prime} .55 \pm 0.029$ & $"$ \\
\hline & 1980.963 & $48 . .472 \pm 0.277$ & $366^{\prime \prime} 182 \pm 0.250$ & USNO A 2.0 \\
\hline & 1990.720 & $48^{\prime \prime} 187 \pm 0.332$ & $366^{\prime \prime} 391 \pm 0 . \prime 300$ & GSC 2.2.1 \\
\hline & 1991.25 & $48^{\prime \prime} 443 \pm 0 . \prime 025$ & $366^{\prime \prime} 006 \pm 0 . \prime 024$ & SPM \\
\hline & 1991.74 & $48^{\prime \prime} 365 \pm 0 . \prime 026$ & - & Tycho 2 \\
\hline & 1991.54 & - & $366^{\prime \prime} 137 \pm 0 . ' 031$ & $"$ \\
\hline & 1999.349 & $48{ }^{\prime \prime} 161 \pm 0 !^{\prime} 009$ & $366^{\prime \prime} 319 \pm 0.021$ & UCAC1 \\
\hline & & $350^{\circ} 45^{\prime}$ & $-06^{\circ} 35^{\prime}$ & \\
\hline \multirow[t]{11}{*}{ HD 220338} & 1892.265 & $17^{\prime \prime} 865 \pm 0.227$ & $42^{\prime \prime} 59 \pm 0 .{ }^{\prime} 02$ & AC2000.2 \\
\hline & 1951.593 & $17^{\prime \prime} .420 \pm 0.252$ & $43^{\prime \prime} 411 \pm 0.250$ & USNO A 2.0 \\
\hline & 1960.84 & $17{ }^{\prime \prime} 687 \pm 0 . \prime 01$ & - & PPM South \\
\hline & 1961.56 & - & $433^{\prime \prime} 329 \pm 00^{\prime} 01$ & $"$ \\
\hline & 1970.484 & $17{ }^{\prime \prime} 666 \pm 0.071$ & - & $\mathrm{CPC} 2$ \\
\hline & 1970.422 & - & $43{ }^{\prime \prime} 438 \pm 0.04$ & $"$ \\
\hline & 1980.781 & $17^{\prime \prime} 716 \pm 0.059$ & $433^{\prime \prime} 566 \pm 0.019$ & TAC 2.0 \\
\hline & 1989.180 & $17^{\prime \prime} .626 \pm 0.300$ & $43^{\prime \prime} 674 \pm 0.300$ & GSC 2.2 \\
\hline & 1991.81 & $17^{\prime \prime} 650 \pm 0.015$ & - & Tycho 2 \\
\hline & 1991.72 & - & 43". $594 \pm 0$ 0'018 & $"$ \\
\hline & 1997.79 & $17^{\prime \prime} 624 \pm 0 . ' 06$ & $433^{\prime \prime} 440 \pm 0.06$ & $\mathrm{CMC}$ \\
\hline
\end{tabular}

